Des voyages de Cook à l'expédition Santo 2006 : un renouveau des explorations naturalistes des îles $d u$ Pacifique

Philippe Bouchet, Hervé Le Guyader et Olivier Pascal

\title{
CpenEdition
}

Journals

Édition électronique

URL : http://journals.openedition.org/jso/4622

DOI : $10.4000 /$ jso.4622

ISSN : 1760-7256

Éditeur

Société des océanistes

Édition imprimée

Date de publication : 15 décembre 2008

Pagination : 167-186

ISBN : 978-2-85430-012-3

ISSN : 0300-953x

Référence électronique

Philippe Bouchet, Hervé Le Guyader et Olivier Pascal, « Des voyages de Cook à l'expédition Santo 2006 : un renouveau des explorations naturalistes des îles du Pacifique », Journal de la Société des Océanistes [En ligne], 126-127 | Année 2008, mis en ligne le 15 décembre 2011, consulté le 17 juin 2020. URL : http://journals.openedition.org/jso/4622 ; DOI : https://doi.org/10.4000/jso.4622 


\section{Des voyages de Cook à l'expédition Santo 2006 : un renouveau des explorations naturalistes des îles du Pacifique}

par

Philippe BOUCHET* ${ }^{*}$, Hervé LE GUYADER** et Olivier PASCAL***

\section{RÉSUMÉ}

Les îles du Pacifique sont célèbres pour le très haut niveau d'endémisme et la grande vulnérabilité de leurs faunes et de leurs flores. Bien que la valeur scientifique et patrimoniale des écosystèmes du Vanuatu ait conduit à classer ce pays comme " point chaud» de la biodiversité, l'archipel souffre d'un déficit de connaissances en comparaison avec d'autres archipels du Pacifique Sud. L'expédition Santo 2006 sur l'île d'Espiritu Santo a eu pour but d'inventorier les faunes et les flores marines, terrestres et dulçaquicoles de cette île, en portant un accent particulier sur les compartiments négligés de la biodiversité. Tous les milieux, depuis les grands fonds marins au large de l'île jusqu'aux récifs, grottes, rivières, forêts et montagnes ont été inventoriés. Des échantillons de tissus biologiques, des enregistrements de sons et des photos numériques des organismes vivants ont complété les prélèvements d'organismes complets. Une attention particulière a été portée aux espèces introduites et invasives, afin d'établir un état de référence de la biodiversité de Santo en ce début du XXI ${ }^{e}$ siècle. L'expédition a impliqué sur le terrain plus de cent cinquante scientifiques, bénévoles et étudiants de vingt-cinq pays.

MoTS-CLÉS: biodiversité, endémisme, découverte, accès, perception

\begin{abstract}
The islands of the Pacific are famous for the high levels of endemism of their flora and fauna, and also the high levels of threats on their faunas and floras. Although Vanuatu is ranked as a biodiversity "hotspot" under many global analyses, the country suffers from a deficit in scientific knowledge on its biota compared to other South Pacific island groups. The goal of the Santo 2006 expedition on the island of Espiritu Santo was to document the marine, freshwater, and terrestrial species of plants and animals living on or around the island, with a focus on the neglected components of biodiversity. All major environments have been sampled, from deep offshore sea bottoms to coral reefs, caves, rivers, forests and mountains; tissue clips, sound records and digital images supplement samples of dried or preserved specimens. In order to establish a baseline inventory of the biodiversity of Santo, special attention was given to introduced and invasive species. Field work involved over 150 scientists, volunteers and students from 25 countries.
\end{abstract}

KEYwORDS: biodiversity, endemism, discovery, access, attitude

\footnotetext{
* Muséum national d'histoire naturelle, pbouchet@mnhn.fr

** Université Pierre et Marie Curie, herve.le_guyader@UPMC.fr

*** Pro-Natura International, ol.pascal@freesurf.fr
} 
La découverte des faunes et des flores du Pacifique n'a pas commencé avec Cook. Les premiers explorateurs Lapita, 1500 ans av J.-C., ont sans aucun doute découvert, à leur manière, les plantes et les animaux des nouveaux territoires qu'ils colonisaient (Galipaud et Lilley, 1999 ; Kirch, 2000 ; Noury, 2005). Mais, s'il n'est pas le premier Européen à explorer l'océan Pacifique, c'est bien avec James Cook (1728-1779) que débute la description scientifique de la biodiversité, au sens où nous utilisons ce mot aujourd'hui. Lors de son premier voyage sur l'Endeavour (17681771), onze scientifiques l'accompagnent (Dumont d'Urville, 1834-1836; Domeny de Rienzi, 1836-1837). Bien que les buts du voyage relèvent principalement de l'astronomie (observation du passage de Vénus le 9 juin 1769) et de la géographie (recherche du continent mythique de la Terra australis), Cook accepte l'embarquement de trois botanistes, Daniel Solander, Herman Spöring et Joseph Banks (1743-1820). La présence de ce dernier revêtira une importance particulière à travers sa qualité de scientifique, mais aussi de gestionnaire, car il deviendra, en 1778, le président de la Royal Society (O'Brian, 1997). Au cours de ses deux premiers voyages - le deuxième ayant eu lieu sur la Resolution et l'Adventure de 1772 à 1775 -, Cook établit l'essentiel de la carte moderne du Pacifique Sud et les naturalistes découvrirent l'extraordinaire biodiversité des îles. À la même époque, la France armait l'expédition de Louis Antoine de Bougainville (1729-1811) qui, sur La Boudeuse et l'Étoile, abordait au Vanuatu en 1768 (Bougainville, 1771). Le botaniste embarqué, Philibert Commerson (1727-1773), décrivit en particulier la bougainvillée (Autheman et Dethorey, 1999). Ces voyages furent à l'origine de nombreuses expéditions qui, au cours de ces deux derniers siècles, amenèrent des naturalistes sur les îles du Pacifique. La manière d'aborder les îles marqua une évolution sensible du $\mathrm{XIX}^{\mathrm{e}}$ au $\mathrm{Xx}^{\mathrm{e}}$ siècle. $\mathrm{Au} \mathrm{XIX}{ }^{\mathrm{e}}$ siècle, les collecteurs sont des religieux, des administrateurs ou des officiers de marine, rarement des scientifiques. Ils tentent de récolter « un peu de tout » partout où ils passent. Le résultat de ces prélèvements est un échantillonnage superficiel des organismes les plus visibles, c'est-à-dire les plus grands ou les plus communs, en général des mammifères, des oiseaux ou des poissons, quelques reptiles. À partir des années 1870 , les collecteurs sont des spécialistes d'un groupe particulier (plantes, papillons, gastéropodes, oiseaux, poissons...), qui vont alimenter en échantillons les zoologistes et les botanistes « de cabinet » qui, pour l'essentiel, ne se rendent jamais sur le terrain. Cette situation change peu à peu dans la première moitié $\mathrm{du} \mathrm{Xx}^{\mathrm{e}}$ siècle avec les expéditions naturalistes généralistes mises en œuvre par de grandes institutions scientifiques proches du Pacifique (Bernice P. Bishop Museum à Honolulu, Australian Museum à Sydney), européennes ou nordaméricaines dans le Pacifique Sud. Malgré les passages à Santo de la Whitney South Sea Expedition (1926) de l'American Museum of Natural History (New York), de l'Oxford University Expedition to the New Hebrides (1933), de la Royal Society-Percy Sladen Expedition to the New Hebrides (1971), les îles des « NouvellesHébrides » restent des cibles scientifiques secondaires par rapport à la Nouvelle-Calédonie ou aux îles Fidji. De ce point de vue, l'expédition Santo 2006 peut donc être considérée comme représentative à la fois d'une continuité et d'un renouveau.

Les îles ont toujours fasciné les naturalistes. Elles continuent de susciter l'intérêt des biologistes contemporains. En effet, les communautés biologiques insulaires paraissent être des écosystèmes plus simples, contenant un nombre plus limité d'espèces que les écosystèmes continentaux : elles présentent à la fois le foisonnement d'espèces des écosystèmes tropicaux et l'appauvrissement biologique propre aux milieux insulaires (Keast et Miller, 1996). L'isolement géographique et écologique de ceux-ci est, en effet, un facteur d'évolution et de spéciation, ainsi qu'un facteur de vulnérabilité : les îles constituent des réservoirs particulièrement riches en espèces endémiques, ainsi que des microcosmes menacés par les introductions d'espèces envahissantes. À ce jour, $72 \%$ des extinctions recensées par l'Union mondiale pour la conservation de la nature (UICN) dans cinq groupes animaux (mammifères, oiseaux, amphibiens, reptiles et mollusques) concernent des espèces insulaires (Baillie et al., 2004).

Les naturalistes se trouvent ainsi, en ce début de $\mathrm{XXI}^{\mathrm{e}}$ siècle, dans une situation paradoxale. D'un côté, ils ont, au cours des vingt dernières années, pris conscience de l'immensité de la magnitude de la biodiversité et sont passés de la proposition :

« Nous connaissons 1,6 millions d'espèces et il en reste peut-être encore autant à découvrir. »

à celle-ci :

« Nous connaissons 1,8 millions d'espèces, mais le nombre réel se situe probablement entre 8 et 30 millions. » (WCMC, 2000)

Les espèces reconnues et nommées représenteraient donc entre 5 et $20 \%$ du nombre réel d'espèces (Bouchet, 2007). D'un autre côté, le 
changement climatique et l'érosion de la biodiversité sont au cœur des préoccupations sociétales sur l'environnement. On ne sait pas si c'est « le quart ou la moitié » des espèces qui pourrait avoir disparu « d'ici le milieu ou la fin du siècle », mais la crise de la biodiversité est une notion qui n'est plus contestée (Leakey and Levin 1995 ; Thomas et al., 2004 ; Pimm et al., 1995 ; Koh et al., 2004) et, pis, qui suit un cours apparemment inéluctable.

Le paradoxe est que, malgré l'ampleur des enjeux, le rythme d'exploration et de description de la biodiversité est dérisoire. Au rythme actuel de la progression des connaissances, il faudrait 250 à 1000 ans pour aboutir à l'inventaire de la biodiversité réclamé par les décideurs, les scientifiques et les gestionnaires. C'est d'ailleurs ce que la Convention sur la diversité biologique (CDB) appelle le «handicap taxonomique» (http://www.cbd.int/gti/; voir, par exemple, Giangrande, 2003 ; Dubois, 2008).

Un autre handicap s'abrite derrière les chiffres vertigineux de cette réalité. Il est rarement exprimé, voire soigneusement masqué tant il gêne les stratégies de ceux qui « vendent» de la « nature » : l'essentiel des zones blanches sur la carte de la biodiversité concerne des animaux peu ou pas attractifs, pour la plupart des invertébrés (crustacés, insectes, acariens, mollusques) qui contiennent les plus forts effectifs d'espèces méconnues au sein de la biodiversité. Or, il semble difficile de provoquer de l'empathie pour un charançon, un ver de terre ou une bactérie. La recherche fondamentale sur ces compartiments négligés de la biodiversité souffre traditionnellement d'un déficit d'attention de la part du public. Celui-ci entraîne indirectement et « involontairement » des difficultés pour obtenir les moyens nécessaires pour les étudier (New, 1995 ; Wheeler and Cracraft, 1997).

Accélérer l'exploration de la biodiversité tout en surmontant ces handicaps constitue donc le grand défi des naturalistes modernes. Nous pensons que les réponses à ce défi se situent dans un changement d'échelle des prospections dans leur ensemble et de méthodes de travail des spécialistes de toutes disciplines participant à celles-ci. C'est ce que le Muséum (MNHN) et Pro-Natura (PNI) ont commencé à faire, chacun de leur côté, depuis une dizaine d'années et en commun, avec l'IRD, au Vanuatu dans l'expédition Santo 2006.

\section{Pourquoi le Vanuatu, pourquoi Santo ?}

Santo (ou Espiritu Santo) est la plus grande île du Vanuatu : $3677 \mathrm{~km}^{2}$, trois fois Tahiti ou la moitié de la Corse, pour seulement 30000 habitants et... une quarantaine de langues ! Santo est aussi l'île la plus élevée de tout l'archipel, parcourue par une chaîne de montagnes dont quatre sommets dépassent $1700 \mathrm{~m}$ et culminant au Tabwemasana à $1879 \mathrm{~m}$. Les richesses naturelles de l'île sont résumées par ce commentaire lapidaire du guide Lonely Planet (O'Byrne and Harcombe, 1999) :

« Sparkling blue holes, unlogged rainforests and the world's largest accessible shipwreck. »

Santo avait ainsi « un peu de tout» à offrir à des naturalistes curieux de découvertes: des récifs coralliens, des grottes, des montagnes, des forêts, des rivières, mais aussi des jardins, des forêts exploitées et une pression immobilière croissante sur les côtes. Bref, un condensé de milieux naturels et anthropisés tels que l'on peut en rencontrer sur les grandes îles hautes de Mélanésie. Compte tenu de sa superficie, de son relief et de son âge géologique, Santo était manifestement sous-explorée. Ainsi, une des dernières prospections botaniques de Santo, en 1988, avait révélé six nouvelles espèces d'orchidées (Lewis and Cribb, 1989). Chez les invertébrés terrestres, l'endémisme, estimé à 30 à $50 \%$, y atteint parfois $80 \%$ (escargots). Le Vanuatu est reconnu par Bird Life International comme une Endemic Bird Area (Stattersfield et al., 1998) et constitue, avec les îles Salomon et les grandes îles au nord-est de la Papouasie Nouvelle-Guinée, le East Melanesian Islands hotspot de Conservation International (Mittermeier et al., 2005).

Au-delà de la diversité des milieux à explorer et des taxons à prospecter, les objectifs des différents modules scientifiques étaient sous-tendus par les mêmes grandes questions : quelle est la dimension réelle de la biodiversité dans ses compartiments les plus divers et dans les milieux les plus riches? Quel est le poids des espèces rares dans la composition des peuplements ? Quelle est la dimension spatiale de cette biodiversité ou, en d'autres termes, quelle est la représentativité des sites à l'échelle éco-régionale ? Quelle est l'emprise des espèces invasives dans les écosystèmes locaux?

Le suivi de l'impact du changement climatique sur la biodiversité est un objectif que se donnent nombre de chercheurs scientifiques. Mais comment mesurer les éventuels changements à venir, quand on ne dispose pas de " point zéro », c'està-dire d'un état des lieux initial, crédible ? Il faut reconnaître que bon nombre de collections dans les muséums - y compris le Muséum national d'histoire naturelle - ont une utilité réduite de ce 
point de vue, car les échantillons qui y ont été déposés ne sont pas assez précisément référencés, en particulier sur le plan géographique. Beaucoup de spécimens sont indiqués comme provenant des « Nouvelles-Hébrides » et portent, au mieux, l'indication de l'île de l'archipel où ils ont été prélevés, mais sans plus de précision.

Il importait donc que l'état des lieux qui devait être dressé en 2006 puisse servir de référence pour le suivi à moyen et long terme de l'évolution des faunes et des flores de ces îles, au XXI ${ }^{\mathrm{e}}$ siècle et au-delà. Les changements à venir concerneront au moins autant la raréfaction et la disparition d'espèces indigènes, que l'introduction et l'établissement d'espèces exogènes. Nous avons donc voulu nous attacher autant à inventorier les espèces considérées comme autochtones que les espèces invasives. Un module baptisé « Friches et aliens » (aliens, pour les espèces invasives animales et végétales) a donc tout naturellement trouvé une place au sein de cette expédition naturaliste du début du XXI ${ }^{\mathrm{e}}$ siècle.

Jusqu'à la première moitié du $\mathrm{xx}^{\mathrm{e}}$ siècle, Santo est restée relativement isolée du monde extérieur. Mais pendant la Deuxième Guerre mondiale, devenue base arrière des troupes américaines engagées dans la Guerre du Pacifique, elle a hébergé jusqu'à 250000 soldats us et leurs infrastructures, subissant de ce fait de fortes perturbations anthropiques. Ainsi, les invasions biologiques qu'elle a connues correspondent à plusieurs périodes historiques, que l'on peut résumer ainsi : arrivée des premiers Mélanésiens il y a plus de 3000 ans (Kirch and Hunt, 1997); arrivée des premiers Européens aux $\mathrm{XVIII}^{\mathrm{e}}$ et $\mathrm{XIX}^{\mathrm{e}}$ siècles ; Deuxième Guerre mondiale; dernières décennies qui voient une augmentation importante des échanges dans l'archipel et avec le reste du Pacifique.

Ainsi, au delà d'un inventaire naturaliste « classique », les objectifs de l'expédition Santo 2006 ont compris aussi l'inventaire des espèces allochtones dans un certain groupe de taxons, la comparaison du poids relatif des espèces allochtones dans les peuplements actuels anthropisés et moins anthropisés, la mise en évidence de l'évolution de la fraction d'espèces introduites, ou disparues, depuis l'arrivée de l'homme et, enfin, l'étude de la perception des espèces allochtones par les populations locales.

\section{La préparation de l'expédition}

Une fois constituée l'ébauche du programme scientifique et avant même le bouclage de son budget, une première mission de trois personnes fut conduite au Vanuatu en mars 2005, dans le but de présenter le projet aux autorités politiques et administratives du pays et à l'ambassade de France, et de faire les premiers repérages logistiques à Santo même. Dès cette première visite, Russell Nari - alors à l'Environment Unit du Vanuatu - et Bernard Sexe - de l'ambassade de France - manifestèrent un soutien appuyé au projet, qui ne devait pas faiblir à long terme. Un échange de lettres avec le ministre des Terres marqua le premier engagement formel $\mathrm{du}$ Vanuatu à l'égard de l'expédition.

À partir de là, les événements s'enchaînèrent. Rufino Pineda fut recruté comme représentant du projet à Luganville même, capitale de Santo. Son expérience, sa maîtrise des langues et ses activités dans le domaine du développement rural (plus de vingt-cinq ans en Mélanésie, dont vingt ans au Vanuatu, responsable en 2003-2005 du programme de reforestation LEARN financé par l'Union européenne) ont facilité son recrutement comme coordinateur local. De juillet à novembre 2005, d'autres missions de repérage affinèrent les objectifs des modules " karst » et " forêts, montagnes et rivières ». Il était prévu, pour ce dernier module, d'établir un camp à proximité d'une forêt aussi « primaire " que possible (et donc sans doute loin des zones habitées), avec le triple objectif suivant :

a. servir de camp de passage pour des petits groupes de scientifiques itinérants,

b. permettre l'étude des variations de la biodiversité végétale et animale le long d'un gradient d'altitude (programme IBISCA),

c. servir de base opérationnelle et expérimentale pour un nouvel aérostat destiné à l'étude de la haute canopée : l'arboglisseur.

Un repérage aérien ayant montré l'intérêt potentiel, pour l'expédition, de la côte ouest du Cap Cumberland, trois sites potentiels y furent visités en novembre 2005 : Tasmate, Penaoru et Olpoi. Dans chaque communauté, nous avons présenté le projet en indiquant que nous étions à la recherche d'un site où nous voudrions étudier la végétation et la faune locales. La forêt de kaoris au-dessus du village paraissant le meilleur choix, nous avons conclu que Penaoru serait le site idéal. Lors de notre deuxième réunion avec le chef Wortut, celui-ci nous a informés que l'ensemble du village dont il se faisait le porteparole était très favorable à l'accueil de l'expédition Santo 2006. Nous avons alors jeté les bases de l'accord qui allait lier la communauté de Penaoru et le projet. Cet accord a inclus, en particulier, la construction, à une heure de marche en amont du village de Penaoru, sur la rivière éponyme, du camp de base, comprenant huit cases d'habitation en bambou sur pilotis, un 


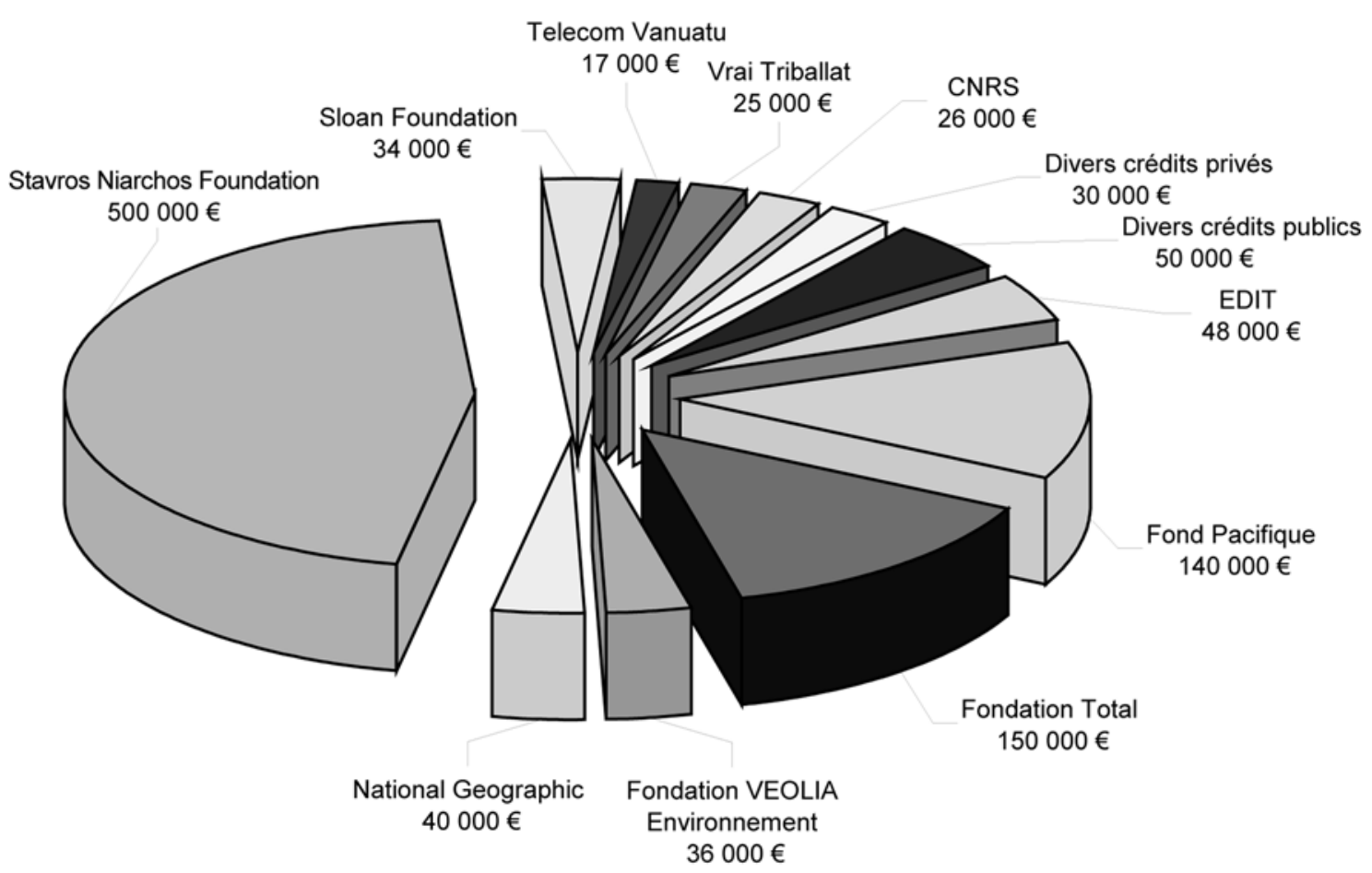

FIGURE 1. - Origine des financements pour le budget opérationnel de l'expédition Santo 2006

réfectoire, une case «cuisine», deux cases "laboratoire», une case "magasin de stockage », deux toilettes, un terrain d'atterrissage pour l'arboglisseur et un pont pour enjamber la rivière. Un devis fut établi pour chacun des bâtiments, dont la plupart devaient être édifiés en matériaux de construction locaux (bois, bambou, feuilles de natangora), l'expédition prenant en charge par ailleurs l'achat des matériaux non disponibles sur place tels que clous, bâches en plastique... Le coût de la construction du camp fut estimé d'un commun accord entre les deux parties, avec un engagement contractuel auquel s'ajoutait un éventuel bonus de «bon achèvement ».

À Luganville même, les infrastructures existantes rendaient plus faciles l'installation de lieux de vie et de travail pour les scientifiques de l'expédition. Les directeurs du Vanuatu Maritime College (vMc) et du Centre technique de recherche en agronomie du Vanuatu (CTRAV) ont donné leur accord pour l'installation des modules « biodiversité marine» (VMC), "karst» et « friches et aliens » (CTRAV) dans leurs locaux. En France, les trois organismes - Muséum national d'histoire naturelle, Institut de recherche pour le développement, Pro-Natura International - se lient par une convention tripartite. Un comité directeur, constitué par les trois signataires du présent article, est formé, de même qu'un comité scientifique, composé des responsables des grands « modules ».

Une nouvelle mission, en novembre 2005, aboutit à une ébauche de convention (Memorandum of Understanding) entre le projet et les autorités locales. Dans les semaines qui suivent, cette ébauche de convention circule dans les services des administrations à Port-Vila mais des réserves sont émises sur certains des objectifs de l'expédition dans le domaine de l'ethnobotanique - en particulier de l'ethnopharmacologie. Dans la mesure où cet objectif était très mineur (un seul chercheur) dans notre projet, et pour ne pas être suspectés de biopiraterie, nous décidons de supprimer ce thème de recherche. La convention est signée le 24 mars 2006 par le ministre des Terres, Maxime Korman Carlot, et le directeur du Muséum, Bertrand-Pierre Galey, représentant Santo 2006. À son tour, la convention ouvre la voie à un permis de recherche, délivré collectivement à l'ensemble de l'expédition, signé le 2 juin 2006 par Ernest Bani, le directeur de l'Environment Unit.

Pendant ce temps-là, les bonnes fées des fondations, entreprises et financements publics penchées sur le berceau de l'expédition, avaient permis de recueillir 1,1 million d'euros d'aides publiques et privées. Au premier rang de ces institutions figurent la Fondation Stavros Niarchos, la Fondation Total et le Fonds Pacifique 
(figure 1). Nos salaires et les aides en nature venant de nos trois organismes de recherche ne sont pas pris en compte dans le budget de l'expédition. Le déploiement de l'Alis, le navire de recherche de l'IRD, valut à lui seul près de $250000 €$ mais ne fut jamais, lui non plus, comptabilisé dans le coût de l'expédition. De même, le camp de Penaoru bénéficia gracieusement de la mise en place d'une antenne satellite par Telecom Vanuatu.

Ce budget ne prend pas en compte, non plus, les coûts liés à la communication et aux médias (film, site web, presse), couverts par les éditeurs, producteurs et diffuseurs concernés.

Localement, nous savions qu'un accord du ministre à Port-Vila, ou du secrétaire de Province à Luganville, était indispensable, mais non suffisant. À Santo, comme dans le reste du Vanuatu, le vrai pouvoir d'accueillir est au niveau des communautés elles-mêmes, au niveau de la coutume et des villages. Nous avons donc dû communiquer avec tous les membres de ces communautés et les informer de nos projets, avec le concours des chefs coutumiers, des field workers du Vanuatu Kaljarol Senta (vKs) et du député de ouest Santo, Sela Molissa, qui s'est personnellement investi dans la sensibilisation des habitants de « sa » circonscription. Une grande réunion eut lieu à Luganville au nakamal des chefs, dans les locaux de la province de Sanma, au cours de laquelle les objectifs de l'expédition firent l'objet d'une présentation en anglais et en bislama, devant plusieurs dizaines de participants venus tout exprès de Sud Santo, Kerepoa, Malo, Aoré, Tutuba, Port Olry... Le Rotary Club de Santo, le Tourist Board..., personne ne fut oublié. En août 2006, tout était donc prêt pour le démarrage de l'expédition.

\section{Le déroulement de l'expédition}

Les premiers scientifiques arrivent en petit nombre en août 2006 (figure 2). Malgré les missions de repérage de 2005 et 2006, nous avions surestimé l'adéquation des moyens locaux à nos besoins et sous-estimé les lenteurs tropicales de toutes sortes. Toutes les expéditions de fret sont arrivées avec trois à huit semaines de retard et nous avons donc dû modifier le calendrier des premières arrivées. Le premier mois devait être un mois de démarrage avec des effectifs réduits : il a effectivement rempli ce rôle et les tout premiers participants arrivés au mois d'août (le groupe d'algologues de Claude Payri, de Nouméa, et le petit groupe de Rapid Assessment avec Fred Wells, de Perth) ont dû faire face aux pre- mières difficultés : pluies diluviennes (août est pourtant statistiquement le mois le plus « sec » de l'année!), transformation inachevée du hangar à bateau en "laboratoire» marin, ennuis mécaniques sur les petits bateaux du vMC, défauts de communication avec certains propriétaires « riverains » du canal du Segond... Bref, l'expédition se met en place. Fin août, Philippe Bouchet est rejoint par le petit groupe de Philippins avec lesquels il avait monté Panglao 2004 et par un volontaire venu de métropole. Tous font alors merveille pour finaliser l'organisation $\mathrm{du}$ laboratoire et des « services » extérieurs (aire de tamisage, emplacement pour le compresseur, tables de tri) et s'assurer que les services domestiques seront au point lors des arrivées massives de septembre.

Du côté marin, nous n'avons pas été épargnés par les péripéties, la dernière étant la mise en grève, pour des raisons liées à un changement de statut, d'une partie de l'équipage de l'Alis au moment de l'appareillage de Nouméa, le 6 septembre. Avec l'arrivée massive du 8 septembre (une soixantaine de personnes), il a fallu monter un plan B impliquant un bateau de la Marine nationale. Le samedi 9 a été un jour d'organisation du chaos et l'échantillonnage a pu commencer le 10. L'Alis arrive finalement en fin de journée, le mardi 12.

Les sorties sur le terrain démarrent à 7 heures, différents bateaux emmenant des groupes de plongeurs, chacun avec un objectif particulier (collecte générale, collecte ciblée de nudibranches, brossage de cailloux, pose de filets maillants...). Au laboratoire, les journées commencent un peu plus tard et se terminent vers 22 ou 23 heures, puisqu'il faut traiter sur le frais les collectes du jour (tri, photo numérique, fixations du matériel destiné au séquençage). La langue de travail est l'anglais, mais on parle ici et là d'autres langues dans de plus petits groupes. En particulier, dans le hangar à bateaux, devenu laboratoire maritime, on parle aussi (ce qui suppose qu'au moins deux personnes parlent une même langue !): chinois, japonais, tagalog, cebuano, suédois, norvégien, italien, espagnol, néerlandais, russe, allemand et, bien entendu, français et bislama. Au total, l'expédition implique des participants originaires de vingt-cinq pays.

Côté karst, l'équipe est au complet, la petite vingtaine de personnes est opérationnelle dès le $1^{\mathrm{er}}$ septembre. Elle fonctionne par petits groupes de trois à sept personnes travaillant en parallèle sur plusieurs sites dispersés à l'est et au centre de Santo et dans les îles proches : les unes recherchent plutôt les abris sous roche secs, d'autres 


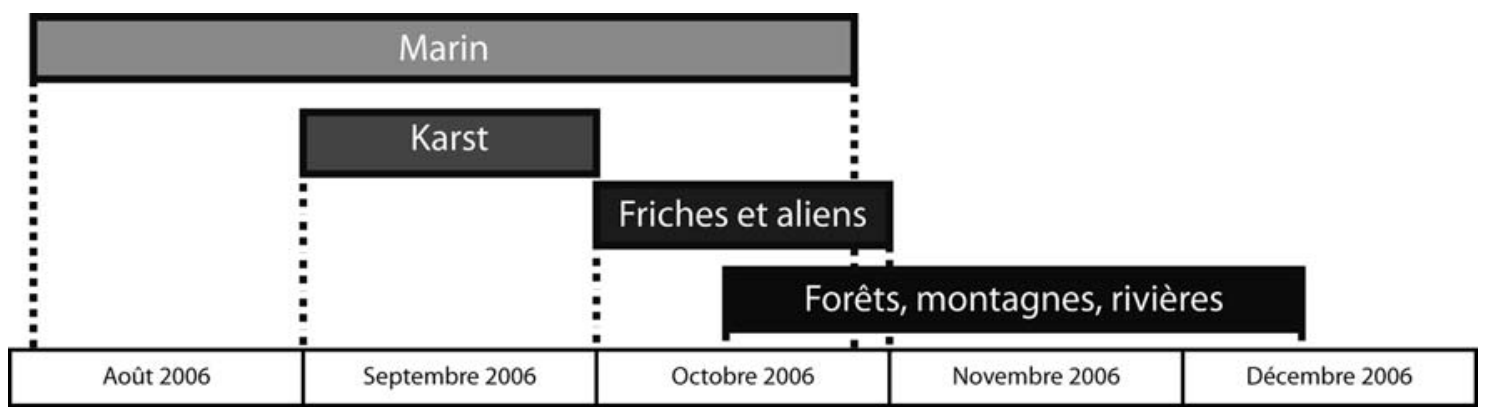

FIgURE 2. - Calendrier de présence sur le terrain des quatre modules de l'expédition Santo 2006

des grottes à guano, d'autres enfin les aquifères souterrains. Un petit groupe explore la pointe du cap Cumberland, une région très isolée (à cinq jours de marche du bout de la route) où les a conduit l'Etelis, petit bateau du Maritime College. Le groupe karst est hébergé au CTRAV à une quinzaine de kilomètres de Luganville et nos chemins se croisent assez peu. Nous échangeons nos impressions, nos humeurs et nos anecdotes lorsqu'ils viennent chez les « marins » s'approvisionner en carburant, en formol et en alcool. Nous organisons donc sur le site du Maritime College deux soirées d' "animation sociale» qui sont l'occasion pour l'expédition d'inviter les personnels des organismes qui nous hébergent, des enseignants et des commerçants de Luganville.

Les péripéties et les aléas se succèdent, heureusement sans blessé, sans dérapage budgétaire et sans affrontements entre participants. Dans le groupe de recherche sur le karst, la semaine du 18 septembre sera marquée par la découverte du plus grand réseau de l'île, avec trois kilomètres de galeries reconnues et topographiées dans le centre de Santo et l'exploration d'un grand puits de 50 mètres de profondeur sur Malo, la plus grande des « petites îles », devant Luganville. Ces cavités étaient en partie connues par les villageois, mais ne nous avaient pas été révélées spontanément dans un premier temps. À mesure que notre équipe explore le terrain, les langues des habitants de Santo se délient. Finalement, la curiosité l'emporte et des propriétaires coutumiers viennent nous proposer de nous montrer une nouvelle cavité. L'équipe qui était partie au cap Cumberland a découvert d'intéressants sites au plan de l'archéologie, mais est revenue bredouille pour ce qui concerne les vertébrés quaternaires. La semaine aura également été marquée par l'arrivée des premiers photographes, cinéastes et journalistes envoyés de France et de Nouvelle-Calédonie par ATOM et Gamma. Compte tenu de l'avancement du module karst, ils ont tout naturellement com- mencé à collaborer avec ce groupe. Franck Bréhier et Nadir Lasson ont été leurs " passeurs » dans les siphons de la grotte de Loren, presqu'au bout du cap Quiros. En marge des grottes, Louis Deharveng n'est pas avare de superlatifs pour qualifier la faune du sol de Santo : le paradis des collemboles!

Dans le domaine marin, le travail a été un peu perturbé par l'alizé qui a soufflé une bonne partie de la semaine (du 18 au 22 septembre 2006) et nous a obligés à nous rabattre sur les côtes les moins exposées. Les plongeurs sont frappés par le fait que de nombreux récifs sont morts, sans qu'on puisse attribuer la cause de cette mortalité à la surpêche (il n'y a pratiquement pas de pêcheurs et pas de pêche à la dynamite, comme aux Philippines) ou à d'autres activités humaines identifiées. Peut-être la combinaison des cyclones, des tremblements de terre (une jolie petite secousse début août et plusieurs autres, insignifiantes, depuis) et du blanchissement (dû à l'élévation de la température de l'eau de mer) est-elle responsable? Malgré la complexité topographique de la zone côtière, les habitats sont relativement peu variés; nous n'avons pas encore trouvé d'herbiers et les mangroves sont restreintes. La semaine aura également été marquée par le démarrage des premières plongées dites « de nuit» (en fait, la nuit tombe à 18 heures et les plongées de nuit ont lieu entre $19 \mathrm{~h} 30$ et $22 \mathrm{~h}$ ). Le dimanche 24 septembre est le premier jour de repos. La saison des agrumes s'achève et les manguiers sont en fleurs.

$1^{\text {er }}$ octobre 2006. Les spéléologues du karst laissent la place aux biologistes de " friches et aliens », tandis que les effectifs des biologistes marins restent soutenus. Un peu plus de la moitié des participants à Santo 2006 est à Santo ou en est même déjà repartie; seul le gros chantier «forêts, montagnes, rivières" n'a pas encore démarré, mais les préparatifs du camp de Penaoru avancent normalement.

La fatigue et le climat font un peu sentir leurs effets sur les personnes et le matériel. Petits 
bobos ou fièvre ont retenu cette semaine plusieurs d'entre nous quelques jours hors de l'eau et éloignés des paillasses d'observation. Une morsure de murène vaudra cinq points de suture à son bénéficiaire. Un autre chercheur sera victime d'une morsure de scolopendre. Du côté du matériel, on relève une cassure d'essieu sur un véhicule, une autre d'arbre d'hélice sur un des bateaux qui sera hors service jusqu'à la fin de la mission.

Dans le module karst, la loi de Murphy est vérifiée une fois de plus. La semaine précédente avait été marquée par la découverte du plus grand réseau de l'île; record battu la dernière semaine de septembre, avec la mise au jour d'un nouveau réseau et de galeries gigantesques, découvertes quelques jours avant le départ et qu'il n'aura pas été possible d'explorer complètement. Ces nouveaux réseaux paraissent recéler des organismes symptomatiques de la faune souterraine cavernicole, alors que les grottes explorées jusqu'à présent étaient essentiellement habitées par la même faune que celle des sols de surface. Biologiquement, les eaux souterraines de Santo se sont globalement révélées très pauvres, ce qui doit refléter d'une part l'âge géologique récent de cette partie de l'île, d'autre part le régime torrentiel de circulation des eaux. Cette observation vaut aussi du côté des vertébrés quaternaires. Par contre, et cela fournit de grandes satisfactions, Louis Deharveng quitte Santo avec une centaine environ d'espèces nouvelles de collemboles.

Dans le domaine marin, nos sites d'échantillonnage se sont appelés, cette semaine, Pallicolo, Surunda, Aesa, Mavéa, Baie de la Tortue ou encore Oyster Island. Nous avons basé nos petits bateaux à une trentaine de kilomètres du laboratoire, ce qui a permis de gagner du temps de transit pour les plongeurs mais a nécessité un grand nombre de transbordements, par la route, de bouteilles, bacs, paniers et appareils. Quelques beaux récifs et tombants s'observent sur cette côte Est et une collecte d'espèces qui ne faiblit toujours pas. Depuis samedi, il pleut mais, au moins, le vent est tombé et nous pensons consacrer la semaine qui vient aux côtes les plus exposées, au droit des localités de Tutuba, Malo et Urelapa.

Le jeudi 5 octobre était fête nationale au Vanuatu et nous avions, de notre côté, déclaré le 6 journée Portes Ouvertes sur notre site du Maritime College. Nous avions invité directeurs d'écoles, professeurs de sciences, officiels de la
Province et chefs coutumiers à visiter notre installation, à venir nous poser des questions, à regarder des animaux à la loupe binoculaire... Après quelques petits ratés au démarrage, en août, nos rapports avec les autorités et la population étaient devenus excellents et plus personne apparemment ne nous soupçonnait d'être là pour piller les «ressources génétiques » du Vanuatu. Le Daily Post, le seul quotidien du pays, consacre régulièrement une pleine page à Santo 2006. Bref, notre expédition au long cours est maintenant entrée dans l'actualité ordinaire de Santo.

La lune était pleine ce 7 octobre et cette semaine était donc une semaine de grandes marées à Santo. Nous avons profité de ces bonnes marées pour passer au peigne fin un platier en apparence banal, devant lequel nous passions presque tous les jours à la sortie de la ville, juste à côté du dépôt de carburant ! Une découverte sensationnelle nous y attendait. Nos collègues japonais Yasunori Kano et Takuma Haba ayant eu, en effet, la curiosité de creuser sous de gros blocs dans une résurgence d'eau douce recouverte par la mer à marée haute, ont mis la main sur une petite faunule entièrement nouvelle de gastéropodes.

Si la première semaine d'octobre a été marquée par les grandes marées, la deuxième devait l'être par les plongées très profondes. En effet, notre dream team de plongeurs au mélange trimix $^{1}$ est arrivée d'Honolulu, tous les problèmes de raccords et de valves ont été surmontés. L'équipe de Richard Pyle peut ainsi s'attaquer aux récifs profonds de Tutuba, jusqu'à 120 mètres de profondeur. Avec des temps de décompression qui se chiffrent en heures, la patience de Jean-François Barazer, qui assure la sécurité en surface, se mesure, elle, en gros livres et en recueils de sudokus.

Nous continuons à connaître un bien mauvais temps. Nous plaignons d'avance les botanistes et les entomologistes qui vont travailler dans des forêts ruisselantes avant de passer leurs nuits en bivouac. Avec Laurent Soldati, de l'INRA, arrivé depuis une semaine, Hervé Jourdan, de l'IRD, et le groupe de Laure Desutter qui arrive à Santo dans quelques jours, la recherche entomologique va maintenant monter en puissance dans Santo 2006. De même, les effectifs, encore réduits au seul Marc Pignal, de l'équipe botanique vont également se renforcer.

15 octobre 2006. Le module «friches et aliens » a rempli déjà la moitié de son pro-

1. Le trimix est un mélange judicieusement dosé de trois gaz (oxygène, azote et hélium), qui permet, sous forte pression, d'éviter, d'une part, une hyperoxie qui peut conduire à des crises convulsives et, d'autre part, la narcose (ivresse des profondeurs) due à l'azote. 
gramme. L'objet même de ce module étant l'échantillonnage de sites anthropisés, ses terrains sont évidemment moins naturels que ceux du module karst : zone portuaire de Luganville, plantations de cocotiers, caféiers et cacaoyers, jardins mélanésiens. Un des premiers résultats alarmant qu'ils dégagent est que la fourmi électrique Wasmannia auropunctata est déjà arrivée sur Santo, apparemment à partir d'échanges de plants avec les îles Banks, foyer infesté par la fourmi depuis plusieurs années. Partout où elle a été introduite dans les îles du Pacifique, cette fourmi américaine cause de gros dégâts écologiques et se révèle être une gêne considérable pour les humains et les animaux domestiques. Pour le moment, il semble que l'infestation à Santo soit limitée à moins d'un hectare, ce qui la rend encore jugulable, et Hervé Jourdan, « Monsieur Fourmi électrique », à l'IRD, est en train de mettre en place un plan d'éradication. D'autres espèces de fourmis introduites sont présentes à Santo, mais Hervé est surpris de constater qu'à la différence de la Nouvelle-Calédonie, elles ne cohabitent finalement pas trop mal avec les fourmis locales.

À l'exception des chauves-souris, toutes les espèces de mammifères sauvages de Santo ont été introduites. On peut attribuer aux premiers Mélanésiens la présence du rat du Pacifique (Rattus exulans), du porc marron et du chien marron, aux Européens celle du rat noir (Rattus rattus) et du rat surmulot (Rattus norvegicus), de la souris grise, du bœuf marron et du chat marron. Des restes du grand rat épineux de Nouvelle-Guinée (Rattus praetor) ont été trouvés dans des sites archéologiques.

Deux espèces végétales envahissantes, Merremia peltata (Convolvulacée) et Mikania micrantha (Astéracée), ont fait l'objet d'observations détaillées de la part de Marc Pignal. Merremia est réputée être hautement invasive, mais elle a un nom dans la langue de Butmas, village du centre de Santo, et se trouve dans sa zone de répartition naturelle. Il est vraisemblable que son comportement agressif (dès l'apparition d'une trouée de lumière, elle émet des tiges verticales qui envahissent les arbres et les tuent) soit une conséquence de l'exploitation forestière anarchique. Mikania est originaire d'Amérique tropicale, d'où son nom de liane américaine. La petite histoire locale attribue cette introduction à l'armée américaine, qui aurait utilisé celle-ci au cours de la Deuxième Guerre mondiale pour le camouflage des installations. Si cette histoire est probablement fausse, tout laisse penser que l'introduction de la plante date bien de cette époque.
Le premier groupe itinérant «Forêts" est dans le dark bush depuis quelques jours. En dehors d'une vingtaine de kilomètres autour de Luganville, il n'y a que le téléphone Iridium qui permette de communiquer. C'est donc le silence radio avec ce groupe.

Tous les manuels de biologie marine racontent l'histoire du palolo, ces vers qui, une fois par an, se rassemblent pour lâcher leurs gamètes et se reproduire; tous les manuels... mais bien peu d'auteurs de manuels ont vu eux-mêmes le rassemblement de ces vers. En tout cas, aucun des scientifiques de Santo 2006 n'avait vu ce rassemblement. Le phénomène se produit avec une régularité astronomique. À Santo, six jours après la pleine lune d'octobre, nous avons donc eu la chance de vivre l'événement au village de Tongoa pendant la nuit du 12 au 13 octobre. La pêche démarre à la marée descendante, vers 22 heures, et se termine vers 1 heure du matin au lever de la lune. Et, le lendemain, on mange un délicieux laplap au palolo, plat à base de manioc surmonté de vers verts. C'est un peu curieux mais délicieux...

Le groupe de Richard Pyle du Bishop Museum d'Honolulu a plongé cette semaine jusqu'à 140 mètres de profondeur et expérimenté avec succès un nouveau modèle de suceuse jusqu'à 120 mètres. Plusieurs découvertes remarquables sont déjà venues récompenser ces tentatives et la peine que nous avions prise pour faire venir de l'hélium de Nouméa et 300 kilos de fret d'Honolulu.

Des visites de personnalités ponctuent de loin en loin l'expédition. Cette semaine Ham Lini Vanuaroroa, le Premier ministre du Vanuatu, a passé une petite heure avec nous.

22 octobre 2006. Les bruits des activités du module «marin» viennent de s'éteindre. La petite noria de botanistes et d'entomologistes prend la suite. Déjà, le groupe de Laure Desutter et d'Odile Poncy prospecte depuis plus d'une semaine, l'équipe d'Adeline Soulier-Perkins, Ronan Kirsch et Gregory Plunkett embarque demain soir pour s'attaquer au Tabwemasana, le point culminant de Santo, et Bruno Corbara, Jérome Munzinger et Marika Tuiwawa sélectionnent et balisent les sites IBISCA pour décrire les effets du gradient d'altitude sur la flore et les insectes dans la forêt de kaoris, au dessus de Penaoru.

Deux tremblements de terre ont eu lieu cette semaine à Santo. Le premier a atteint 6,2 sur l'échelle de Richter. Son épicentre a été situé à $50 \mathrm{~km}$ de Luganville. Il a duré trente bonnes secondes (juste assez pour intimider et juste assez peu pour ne pas effrayer). Le deuxième a 
été ressenti à Penaoru. En cette troisième semaine d'octobre, l'Euphrosyne a amené par la mer la dernière vague de logisticiens et la première vague de chercheurs. Antenne satellite, générateurs, ordinateurs : le premier coup de téléphone et la première connexion internet ont été établis de ce village où l'on s'éclaire le soir à la lampe à kérosène. L'émotion et l'enthousiasme d'Olivier Pascal se ressentaient lorsqu'il appela Luganville pour décrire tous les villageois agglutinés devant l'ordinateur, regardant leur pays, leur île, leur coin de vallée, sur Google Earth. Petite victoire technologique, mais dont la saveur s'apprécie mieux au récit des tracas des participants au module "forêts ", soumis aux aléas maritimes : à Santo, les dernières forêts se gagnent par la mer.

Pour faire écho aux secousses telluriques, deux cyclones sont venus encadrer le début et la fin des travaux dans les vestiges de forêt naturelle de l'île et compliquer les opérations terrestres. Pas de dégâts physiques, mais le moral et les nerfs ont été sérieusement mis à l'épreuve. Le premier, Xavier, est resté bloqué plusieurs jours à environ 200 miles nautiques au nord-est de l'archipel, empêchant toute navigation aux abords de Santo et forçant le cargo transportant notre conteneur depuis la Nouvelle-Calédonie à faire demi-tour ; épisode fâcheux et irritant, après avoir bataillé avec les transporteurs pour acheminer notre matériel immobilisé à Singapour et pour trouver une route maritime alternative (et d'abord un bateau, rare dans ces parages) pour que les équipements parviennent en temps et en heure à Santo. À 24 heures près, nous aurions profité du passage hebdomadaire de la barge Brisk, seule capable de livrer nos équipements directement sur une plage sans pontons. La conséquence de tout cela fut, pour le camp de Penaoru, un début nettement plus désordonné que prévu... Pendant deux jours, au lieu de terminer l'aménagement, c'est au démontage des toitures et à l'abattage des arbres les plus dangereux que les villageois se consacreront au campement. Le gros des troupes du projet IBISCA ayant débarqué avec l'Euphrosyne avant l'arrivée de la barge de matériel, ils trouveront un laboratoire vide, tout le matériel étant dans les containers... Fort heureusement, tout rentrera dans l'ordre en peu de temps.

L'accès à la côte ouest de l'île n'est pas une partie de plaisir, c'est une côte « lisse » sans barrière corallienne et pratiquement dépourvue de mouillages pour la protéger de la menace aléatoire des «coups d'Ouest». Débarquer les équipes, après 10 heures de mer depuis Luganville, sur la plage de galets à l'embouchure de la Penaoru, par forte houle, n'est pas simple.
Décharger les 10 tonnes de matériels, dont l'arboglisseur et les gaz nécessaires à son fonctionnement (propane et hélium) l'est encore moins. C'est là aussi une affaire de chance : si la houle est trop forte, la barge ne peut s'échouer sur le rivage. La mer ondule légèrement ce matin de 11 novembre, signant notre armistice avec les éléments et les transporteurs maritimes. Aidée par les gens des villages de Penaoru et de Peavot, une chaîne s'organise pour vider au plus vite le pont du Brisk. Une impression de naufrage règne sur la plage, où s'entassent pêle-mêle caisses et conteneurs de toutes sortes. Trois jours seront nécessaires pour transporter à dos d'homme le matériel de la mission sur les quatre kilomètres qui séparent le rivage du camp de Penaoru, situé au fond de la vallée du même nom.

Le deuxième cyclone s'est invité sur le tard, environ trois semaines après Xavier, à la fin de novembre. Les vents violents nous ont contraint à évacuer le « camp 2 » installé sur une crête à $900 \mathrm{~m}$ d'altitude : trop exposé, ce camp léger, servant de relais pour les équipes travaillant entre 1000 et 1500 mètres, point culminant de la zone, est abandonné temporairement pour le camp principal, coincé en surplomb de la rivière Penaoru, mais mieux abrité que le premier. Le camp de base accueillera jusqu'à cinquante personnes par jour dans sa " haute saison ». Bâti sur une ancienne tarodière, offrant un site plat et dégagé, il domine de quelques mètres la Penaoru qui fournit l'eau indispensable pour un camp de cette taille. Si le confort est sommaire, le « restaurant » est pantagruélique. Initiés par les femmes du village, nos mitrons sont passés maîtres dans la cuisson des cochons au contact de pierres chaudes, recette difficile à exporter, mais qui fait encore saliver, longtemps après le retour.

Nourrir beaucoup et bien... voilà le secret d'une expédition réussie. Les statistiques de l'intendance, tenue par Dan Molczadzki, notre responsable de camp, sont éloquentes. Sont passés sous les couteaux de nos cuisiniers, Guillaume Chipy et Luc Zelmat, 5 bœufs, 14 cochons, 4 chèvres, quelque 80 poulets et 4 tonnes de fruits et légumes. Toutes ces denrées, « commandées » six mois à l'avance aux villages de la région pour ne pas provoquer de pénurie dans ces communautés isolées vivant encore largement en autarcie, constituèrent un ressort essentiel aux scientifiques.

Les dernières forêts intactes de Penaoru ne le sont pas par hasard. Les équipes ont gravi, en dénivelé, des dizaines de milliers de mètres cumulés pour travailler, chaque jour, dans les forêts accrochées au relief. En dessous de $600 \mathrm{~m}$ 
d'altitude, la forêt est dégradée et sa composition en espèces appauvrie. La "belle» forêt commence vers 800-900 mètres, et deux heures de marche, environ, sont nécessaires pour se rendre sur les sites retenus, tous les matins. Le kaori (Agathis macrophylla), un conifère, est à cette altitude l'arbre le plus abondant. Les forêts de kaoris ne sont connues que dans deux autres îles au Vanuatu, Anatom et Erromango, où elles sont particulièrement menacées et surexploitées, conférant à celle de Santo un caractère unique dans l'archipel (Mueller-Dombois and Fosberg, 1998).

Aux altitudes les plus élevées, au-delà de $1200 \mathrm{~m}$, apparaît un type différent de forêt, dominée par des arbres appartenant au genre Metrosideros, et avec un jardin de mousses « aqueux » dont l'australienne Elizabeth Brown, spécialiste de ces plantes, eut du mal à se détacher. Si l'installation du camp 2 fut un soulagement pour ceux qui travaillent jusqu'aux crêtes sommitales vers $1500 \mathrm{~m}$, nombreux sont ceux qui font l'aller-retour dans la journée ${ }^{2}$.

Entre les deux cyclones, aucune fausse note donc. Aucun accident ni incidents majeurs. Seul fait notable et à ranger dans les chroniques des péripéties, le bris de l'hélice de l'arboglisseur. Ce n'est pas tant la rupture d'une pale que les tracas induits qui posent problème. Briser une hélice d'un engin n'est pas grave en soi (l'arboglisseur a fait ses premiers vols à Santo et, comme tout prototype, mérite quelques améliorations), mais en trouver une autre pour que cet engin voué à la récolte en canopée, apporté non sans mal sur la côte ouest de Santo, puisse encore servir, est un vrai casse-tête.

Commander par téléphone (depuis la seule " cabine téléphonique » de la région, installée par le projet comme nous l'avons dit plus haut) une hélice faite sur mesure dans les ateliers Duchélices, en France, a déjà pris quelques heures. L'hélice a été moulée selon les cotes d'origine, envoyée à Paris, puis a voyagé dans divers avions jusqu'à Port-Vila, pour être, enfin, dirigée en fret vers Luganville, réceptionnée par Rufino Pineda, mise dans un petit avion spécialement loué pour la circonstance à destination de l'aérodrome de Lajmoli (le seul de la côte ouest, près du village d'Olpoy, à $15 \mathrm{~km}$ à vol d'oiseau de Penaoru); puis récupérée par Faustin (le « second » de Dan Molczadzki ), parti à l'aube à cheval pour rallier
Lajmoli, l'hélice a failli finir son périple dans la mer. Revenant par le même chemin longeant le littoral, mais à marée haute, la monture n'a pas apprécié la traversée d'un bras de mer, propulsant par-dessus tête hélice et cavalier. Faustin a fini le trajet à pied, en tenant le cheval par la bride et l'hélice miraculée sous le bras. Cette aventure aura pris, au total, une semaine.

Les expéditions ne se déroulent pas, la plupart du temps, comme on les imagine, bien que cela arrive parfois. Passer au crible les versants « sous le vent » des massifs montagneux de la péninsule du Cumberland n'était pas suffisant pour avoir un aperçu global de la flore et de la faune des forêts de l'ouest et du nord de l'île. Une traversée d'ouest en est du cap s'imposait pour collecter des spécimens sur les versants «au vent ». Pour les quinze villageois, dix scientifiques « volontaires ", notre médecin et l'équipe de tournage, la gaieté des premières 24 heures a bientôt fait place à un certain abattement : six jours durant, la pluie tomba à seaux sans discontinuer, transformant une marche en montagne en un calvaire aquatique.

Travail difficile, sinon impossible, pour un maigre résultat: les variations dues à l'effet de versant, anticipées, ne furent pas observées. Et mises à part des différences notables dans l'abondance relative des différentes espèces, le cortège floristique apparut sensiblement le même de part et d'autre de la crête. Pas de quoi bouleverser les inventaires et gonfler les collections obtenues dans la vallée de Penaoru. Un boulouk (bœuf retourné à l'état sauvage, particulièrement abondant dans ces forêts même aux altitudes les plus hautes) fera les frais de cette traversée. Une chasse improvisée apportera ainsi quelques kilos de viande à l'équipée, au demeurant courte en vivres. Passablement épuisée mais ravie, la petite troupe débouchera sur la plage du village de Piamatsina, sur la côte est du Cumberland où les attendait l'Euphrosyne pour faire route sur Luganville, son avant-dernier voyage avec les scientifiques de l'expédition Santo.

Le bateau viendra enfin récupérer les derniers naufragés volontaires dans la vallée de Penaoru le 5 décembre. L'Euphrosyne et son équipage auront ainsi passé près de trente jours en mer au total, pour acheminer les vagues de chercheurs qui se sont succédées à Penaoru pour travailler sur la terre ferme.

2. Nul ne se doute à quel point la recherche sur le terrain peut être aussi physique. L'abnégation du chercheur de terrain est ici à souligner. Parler des résultats d'une expédition est ce qu'on nous demande en général et ce qu'un biologiste en particulier consent à raconter. La modestie l'empêche cependant de décrire correctement ce qu'il a enduré pour les obtenir, peut-être parce qu'il n'en a pas lui-même conscience et que, insensible à ce genre de détail, il trouve tout cela très normal. 


\section{Résultats}

Par rapport aux missions naturalistes classiques, mobilisant le plus souvent un à trois chercheurs sur un thème bien précis, Santo 2006 a innové par la diversité des moyens d'échantillonnage mis en œuvre et la dimension des équipes déployées, sur place pendant la mission et au laboratoire après la mission. À l'exception des oiseaux, la découverte de nouvelles espèces était attendue dans tous les groupes animaux et végétaux. Cependant, même quand on est le premier à explorer une grotte, un récif ou la canopée d'un grand kaori de Santo, il est rare qu'on soit en situation de crier immédiatement «eurêka! » au moment de la collecte.

Dans la plupart des groupes d'invertébrés, le nombre d'espèces déjà connues est tout simplement trop élevé pour qu'un spécialiste puisse mémoriser même dix pour cent des espèces de « son » groupe. La plupart du temps, on ne sait pas au moment de la collecte si ce qu'on récolte est nouveau ou déjà connu. Même si tel coléoptère ou tel crabe nous est inconnu, cela ne veut pas dire pour autant qu'il s'agit d'une espèce nouvelle. Une expédition se termine donc avec des bidons remplis de sacs et de piluliers remplis d'alcool ou de formol, des piles de plantes séchées entre des feuilles de journaux, des images et des sons enregistrés sur des ordinateurs, des cartes annotées, des listes de stations, des points GPS... Les caisses ont quitté Santo fin décembre 2006 et sont arrivées au Muséum en mars 2007. La chaîne d'opérations passe à ce moment-là par un tri spécialisé : tous les échantillons doivent être triés par familles, chaque spécimen doit être rapporté à un " événement de collecte " ou à une station, avec sa latitude, sa longitude et son altitude ou sa profondeur. Il va sans dire que ce ne sont pas les mêmes personnes qui sont capables de trier des diptères pris dans un piège de Malaise ou les gastéropodes ramenés dans un prélèvement de suceuse. Le tri est le goulet d'étranglement sur lequel butent bien des projets d'inventaire, car si la phase de terrain est exaltante, le tri méticuleux peut rapidement devenir monotone. Enfin, lorsque tout le matériel d'un groupe donné est entièrement trié par familles, le vrai travail d'étude systématique peut commencer. Il s'appuie à son tour sur un réseau international de spécialistes. La systématique est une branche de la biologie où il y a peu de compétition entre les chercheurs : il y a tant à étudier et nous sommes si peu nombreux! Les taxonomistes forment donc une "Internationale » où chacun a sa micro-spécialité. Ainsi, Roland Gerstmeier, de l'université de Munich, est un spécialiste des Coléoptères de la famille des Cleridae et c'est donc tout naturellement lui qui a étudié les Cleridae de Santo ; Yves Terryn, de Gand en Belgique, est un spécialiste des gastéropodes marins de la famille des Terebridae et c'est donc tout naturellement lui qui a étudié les Terebridae de Santo. Ce sont ces spécialistes et leurs semblables qui, deux, trois ou... dix ans après la collecte $d$ 'un échantillon, sont en mesure de dire: " cette espèce est nouvelle». Les eurêka ! peuvent même être différés pendant plus longtemps. En effet, il existe des familles pour lesquelles il n'y a aucun spécialiste dans le monde. Bien entendu, il serait anti-scientifique de négliger ou de jeter les échantillons de cette famille : ils ont coûté cher à récolter, à trier et à étiqueter et l'on est presque certain de ne pas pouvoir retourner en chercher quand une opportunité s'offrira de les étudier. C'est précisément un des rôles des muséums d'archiver de telles collections... le temps qu'il faut.

$\mathrm{Au}$ moment où nous écrivons ces lignes, l'expédition est terminée depuis vingt mois, les caisses d'échantillons sont en France depuis dixsept mois, le tri spécialisé est achevé pour la faune et la flore marines depuis onze mois et il est en voie d'achèvement pour les insectes ou la faune du sol. Il est donc possible de dresser un bilan des collectes et des observations et de faire état de résultats ponctuels, mais il est encore trop tôt pour dresser un bilan synthétique des résultats.

$\mathrm{Au}$ total, nous pensons avoir reconnu de l'ordre de 400 espèces de phanérogames, 350 espèces de champignons et 10000 espèces d'animaux, essentiellement des invertébrés, bien sûr. Les nombres sont un peu plus précis dans le domaine marin: 1100 espèces de crustacés décapodes, 4000 espèces de mollusques, 650 espèces de poissons. Pour mettre ces nombres en perspective, rappelons que, dans toutes les mers d'Europe, du Spitzberg aux Canaries et de l'Islande à la Méditerranée orientale, on connaît 672 espèces de crustacés décapodes ! Des centaines d'espèces nouvelles - nous en prédisons, avec prudence, 1000 à 2000 - ont sans doute été collectées et, cela, dans presque tous les groupes.

Mais derrière ces nombres se cache une réalité biologique plus complexe. Aussi paradoxal que cela puisse paraître, les scientifiques de Santo 2006 n'ont pas trouvé la faune et la flore de Santo si riche que cela! Sur terre, les forêts d'altitude, au-dessus de 1200 mètres, malgré une apparence visuelle de jungle exubérante, se sont même révélées particulièrement pauvres en espèces d'insectes ou d'escargots. Que ce soit en mer ou sur terre, plus de la moitié des espèces n'a été vue 
qu'une fois ou deux et, à chaque rencontre, en faible nombre. En mer cependant, la diversité des espèces est bien là, mais avec des effectifs extrêmement faibles et des sites d'occurrence très ponctuels; il faut prospecter activement pour découvrir les espèces une par une, l'une après l'autre, loin du foisonnement de vie auquel on s'attend sur les récifs coralliens.

En apparence, Santo 2006 diffère peu d'une expédition naturaliste conduite il y a 50 ou 100 ans mais, en réalité, la nature même des données obtenues sur les échantillons a beaucoup changé. Nous savons aujourd'hui que, dans tous les écosystèmes, la plupart des espèces sont petites et rares et nous déployons en conséquence des méthodes de collecte et de tri appropriées : pièges de Malaise pour les petits insectes volants, par exemple; berlèses pour la faune du sol; suceuse et paniers de brossage pour les invertébrés sessiles des récifs coralliens ; tamisage des résidus jusqu'au tamis à maille de $0,5 \mathrm{~mm}$; tri systématique des échantillons à la loupe binoculaire. Si nous revenons de Santo avec autant d'espèces nouvelles, c'est avant tout parce que nous avons collecté des animaux dans une gamme de taille généralement très mal échantillonnée. Autre différence avec les expéditions naturalistes des générations précédentes : le géoréférencement des échantillons et la collecte d'images et de sons associés. Au moins 3000 espèces de Santo ont été photographiées sur le vivant et les émissions sonores de plusieurs dizaines (orthoptères, chauves-souris) ont été enregistrées, fournissant des caractères taxonomiques qui ne sont évidemment pas accessibles sur les spécimens de collection. Enfin, l'arrivée massive des techniques moléculaires dans la systématique "de tous les jours" nécessite - ou permet, selon le point de vue duquel on se place - la collecte de tissus frais destinés au séquençage. Sur ce plan, la moisson de Santo est exceptionnelle car nos conditions de travail - que ce soit au camp de Penaoru, au Maritime College, ou au CTRAV - ont bénéficié d'un « confort scientifique » que la plupart des missions naturalistes d'exploration nous auraient envié.

Avec 16 kilomètres de galeries nouvellement explorées et topographiées, Santo 2006 aura pratiquement multiplié par dix le réseau souterrain connu de Santo. De nouveaux sites archéologiques ont été découverts mais, malheureusement, aucun site présentant des restes de vertébrés quaternaires comme il en a été découvert sur Efate (Mead et al., 2002). Les carottages dans les zones marécageuses vont permettre des analyses paléoclimatologiques.

\section{Quelles difficultés avons-nous rencontré ?}

Le handicap taxonomique que nous avons évoqué dans l'introduction est aggravé par les modalités nouvelles d'accès à la biodiversité. Que ce soit aux fins de recherche fondamentale ou appliquée, la collecte d'échantillons, leur exportation, ce qu'il est permis d'en faire, tout cela est désormais strictement encadré par des législations qui trouvent leur origine dans la Convention sur la diversité biologique (CDB). Pensant qu'ils s'attireraient ainsi l'écoute des décideurs et des leaders d'opinion, et donc des crédits pour leurs recherches, certains scientifiques se sont faits les hérauts d'une biodiversité « utile », vers laquelle il n'y aurait qu'à tendre la main pour découvrir de nouvelles molécules et de nouveaux gènes. L'évaluation monétaire de la valeur de service des écosystèmes procède de cette même attitude d'esprit.

\section{Les naturalistes sont-ils des biopirates?}

Cette marchandisation de la biodiversité a engendré un sentiment de suspicion, voire même d'hostilité, à l'égard des scientifiques qui se voient aujourd'hui systématiquement suspectés de biopiraterie de la part de juristes et de diplomates des pays en développement, qui ne veulent, en conséquence, prendre aucun risque politique ou économique en autorisant l'exploration de la biodiversité de leur pays. En dehors du camp des taxonomistes, pour lesquels « chaque espèce compte », la biodiversité tend fortement à être assimilée à une somme de " ressources ». La CDB ne parle d'ailleurs pas d'« espèces » mais de « ressources génétiques » et elle a fait naître dans les pays du Sud des attentes et des espérances de revenus, de brevets et de royalties (GomezPompa, 2004), que nous n'hésitons pas à qualifier de déraisonnables. Les scientifiques des pays du Nord - comme nous-mêmes - motivés par une recherche désintéressée, sont suspectés, au mieux, de faire une recherche sans intérêt pour le pays hôte, au pire de mentir et d'être des biopirates déguisés. Il est alarmant de constater que, alors que tout le monde feint de s'inquiéter de l'avenir de la biodiversité et de l'extinction des espèces, le travail d'exploration et de description de la biodiversité est freiné par de nombreuses barrières réglementaires et mentales.

Le 28 juin 2006, le Daily Post de Port-Vila publia un article intitutlé "Scientific research gets first butting from Government institution », dans lequel Ralph Regenvanu, directeur du vKS (Vanuatu Kaljarol Senta), s'inquiète de l'incapacité du Vanuatu à encadrer et à contrôler les 
recherches sur sa biodiversité et, mettant en cause nommément Santo 2006, remarque que le Vanuatu en est réduit à « s'en remettre à la bonne volonté de nos collègues français pour qu'il n'y ait pas de pillage du patrimoine national $»^{3}$ pendant l'expédition. M. Regenvanu suggèrera un moratoire sur toutes les recherches conduites par des étrangers tant que le pays ne se sera pas doté d'un Scientific Research Council.

Nous sommes là au cœur même du malentendu sur la signification de la biodiversité. Pour les systématiciens, la biodiversité représente des millions d'espèces de plantes, de champignons, d'animaux - principalement des insectes et des parasites -, parmi lesquelles une infime partie (bien moins de $1 \%$ ) est cultivée, élevée ou utilisée par l'homme. Pour les ethnobiologistes, les juristes et les économistes, la biodiversité consiste dans les centaines d'espèces consommées, sélectionnées ou utilisées par l'homme, plus quelques autres dont on n'a pas encore trouvé à quoi elles pourraient servir, mais qui sont considérées comme des réservoirs de gènes et de molécules en attente de brevets.

Pour répondre aux craintes de Ralph Regenvanu, nous avons adressé à notre tour, le 8 juillet, un courrier à Russell Nari, devenu entre-temps directeur au ministère des Terres, pour l'assurer des dispositions que nous avons prises pour qu'il n'y ait pas " pillage " des ressources naturelles $\mathrm{du}$ Vanuatu. Nous y rappelons que nous avons entendu les réserves formulées par les autorités de Port-Vila vis-à-vis des recherches en ethnobotanique et en ethnopharmacologie et nous soulignons que le programme scientifique de l'expédition ne contient plus aucun thème qui puisse être assimilé à de la bioprospection pour des gènes ou des molécules d'intérêt commercial. Nous soulignons également que, pour prévenir tout conflit d'intérêt et toute ambiguïté, nous nous sommes abstenus de rechercher des sources de financement auprès de fondations ou de sociétés du secteur privé dont l'activité est liée aux organismes vivants ou à leurs produits dérivés ; c'est ainsi que nous avons volontairement évité de solliciter le soutien d'entreprises dans les filières des semences, des cosmétiques ou de la pharmacie. Nous soulignons encore que la Convention signée entre le gouvernement du Vanuatu et le MNHN anticipe les inquiétudes des autorités locales, puisqu'elle précise que l'expédition «s'engage à ne collecter des données et des échantillons qu'à des fins de recherche universitaire et pour la gestion de la biodiversité », c'està-dire excluant toute « valorisation industrielle et commerciale». Reflet de l'inquiétude de son directeur vis-à-vis des bioprospecteurs, la convention précise même que «le Centre culturel devra donner son accord préalable à toute publication qui contiendrait des éléments de savoir traditionnel ou indigène recueillis dans le cadre du projet ».

Enfin, nous faisons remarquer que, malgré l'absence d'un Scientific Research Council, nous avons de nous-même activement recherché et sollicité la participation à l'expédition de chercheurs et de techniciens locaux chaque fois que cela était possible et nous avons, en synergie avec le ministère de l'Éducation, cherché à impliqué des étudiants ni-van. Au total, et malgré la faiblesse évidente des effectifs en collaborateurs locaux dans le domaine de la biodiversité, dix Ni-van (Sam Chanel, Tarere Garae, Anthony Harry, Donna Kalfatak, George Matariki, Vatumaraga Molisa, Charles Tari, Graham Taridia, Emily Tasale, Samson Vilvil-Fare) participeront à l'expédition au sein d'une équipe scientifique. À la suite de l'expédition, Samson Vilvil-Fare a obtenu du territoire de Nouvelle-Calédonie une bourse afin de venir préparer un master à l'université Pierre et Marie Curie. Il aura ainsi les bases nécessaires pour susciter, conduire ou évaluer les opérations de gestion de la biodiversité quand il reviendra dans son pays.

Pour faire suite aux réserves émises à PortVila, nous faisons signer à chacun des participants, avant son arrivée sur le terrain, des «Conditions générales » qui précisent qu' " un permis collectif de recherche et de collecte a été délivré par les autorités du Vanuatu à la mission Santo 2006. Le participant déclare avoir pris connaissance des accords signés entre les organisateurs et les autorités du Vanuatu et s'engage à les respecter $»$.

\section{Que laissons-nous derrière nous?}

Les modules marins et « friches et aliens » ont opéré pour l'essentiel dans la partie « développée » de Santo, tandis que les modules karst mais surtout " forêts, montagnes, rivières » ont travaillé dans des régions faiblement développées, sans infrastructures. Que répondre à la question «éthiquement et économiquement, que laissons-nous derrière nous»? En tant qu'organisateurs du projet, nous avons toujours pris soin, quels que soient nos interlocuteurs au Vanuatu, de préciser nos intentions et nos objectifs et souligné qu'un inventaire scientifique de la biodiversité n'est pas un projet de développe- 
ment. Nous avons cependant, partout où cela était possible et dans le respect de nos engagements vis-à-vis de tous les partenaires, y compris financiers, cherché à agir dans le sens des intérêts à plus long terme du Vanuatu et de l'île d'Espiritu Santo en particulier.

Le splendide isolement du village de Penaoru rendait problématique le transport des personnes et du matériel de Luganville à ouest Santo. Nous avions un temps envisagé la location à un opérateur australien d'un bateau de Port-Vila, mais nous avons finalement choisi de remettre en état, sur le budget de l'expédition, l'Euphrosyne, ancien bateau du résident britannique à l'époque du condominium et aujourd'hui propriété du Maritime College. Ce choix, bien que plus onéreux d'un simple point de vue comptable (il en a coûté $105000 €$, soit $10 \%$ du budget opérationnel total de l'expédition), fut fait pour que l'île de Santo et les communautés du grand nord du Vanuatu (îles Torres, en particulier) bénéficient à plus long terme des retombées de l'expédition. Les rapports d'activité de l'Euphrosyne que nous envoie le vMC nous confortent a posteriori dans notre choix, puisque c'est l'ensemble des communautés de l'île qui bénéficie ainsi des services de ce bateau (formation, transport) et donc, indirectement, de l'investissement consenti par le projet.

Si la présence de l'équipe scientifique n'a pas transformé la vie de Penaoru ou de la côte ouest de Santo, elle a toutefois permis de mettre en avant un des villages (Penaoru), qui a bénéficié de cette circonstance. En confiant la direction du camp à Dan Molczadzki, jeune agronome français parlant le bislama, nous avions l'assurance que son expérience acquise lors de deux années passées avec les communautés rurales de Santo (projet POPACA d'aide aux agriculteurs dans les filières coprah, café, cacao) permettrait d'appliquer un certain nombre de normes éthiques/sociales irréprochables : salaire fixe de $1000 \mathrm{vT} /$ jour par personne, tarif défini par le vKS pour ses fieldworkers et appliqué à tous les intervenants (porteurs, guides, aide-cuisiniers) ; jamais plus de 6 jours de travail/personne/ semaine, en moyenne 3 jours/semaine/personne ; système de rotation pour offrir du travail à un maximum de personnes (notamment pour les aides de camp); système de rotation pour l'approvisionnement en produits frais pour garantir des revenus à un maximum de personnes. Un comité de gestion villageois avait été constitué. Des rencontres régulières (presque quotidiennes) entre Dan et Ruben Boe, secrétaire du comité, ont permis d'apporter une attention particulière aux commentaires et/ou revendications des villageois. La plus grosse tension fut, en réalité, « interne » au sein de nos hôtes, les travailleurs issus des villages voisins craignant que les sommes dues ne leur soient pas reversées par le comité de gestion, constitué uniquement par des habitants de Penaoru. Au total, 96 habitants de la côte ouest de Santo ont " participé » au projet; 44 étaient originaires de Penaoru (le village a 103 habitants, femmes, enfants et personnes âgées inclus), les 52 autres étaient pour la plupart originaires des villages voisins de Nokuku, Petawat, Sulesai et Wunon. L'expédition a injecté directement (construction du camp, portage, aides, approvisionnement frais, guides, etc.) environ $2400000 \mathrm{vT}$ (soit environ $18000 €$ ) dans l'économie du village et des villages avoisinants. Outre, bien entendu, les bâtiments du camp lui-même, du matériel a été laissé sur place à la fin de l'expédition, sous la responsabilité du comité de gestion villageois, notamment deux petites cuves à eau, des containers, de l'outillage. Après l'expédition, Rufino Pineda, avec le soutien du Rotary Club de Santo, est revenu installer une grande cuve à eau de 1100 litres ainsi que des tôles pour la collecte de l'eau de pluie. L'idée, un instant caressée, de transformer le camp en structure d'accueil pour touristes friands d'écotourisme a fait long feu, le propriétaire coutumier du site où était implanté le camp n'acceptant pas de partager avec la communauté la gestion et les bénéfices éventuels retirés de son exploitation.

\section{Épilogue}

L'expédition Santo 2006 a montré que des inventaires ambitieux sont possibles en déployant les moyens humains et logistiques appropriés ${ }^{4}$ (figure 3). À Santo, l'inventaire de la faune et de la flore a atteint un grand degré d'exhaustivité et l'objectif initial d'échantillonner « du battant des lames au sommet des montagnes » a été atteint (figure 4). L'expédition n'était pas la première montée par les trois organismes partenaires du projet, mais elle fut sans aucun doute la première à impliquer autant de scientifiques sur le terrain, à entraîner autant de retombées en communication et, d'une certaine façon, à redorer le blason de l'exploration naturaliste. Que les chercheurs reviennent d'une île

4. Quelques données sur le module marin : les bateaux ont consommé 6800 litres d'essence et parcouru chacun environ 1200 miles ; nous avons gonflé environ 3000 bouteilles de plongée, consommé 5000 repas et réalisé un peu plus de 500 opérations de terrain. Les collections ont absorbé 1000 litres d'alcool et occupent 65 fûts de 40 litres. 


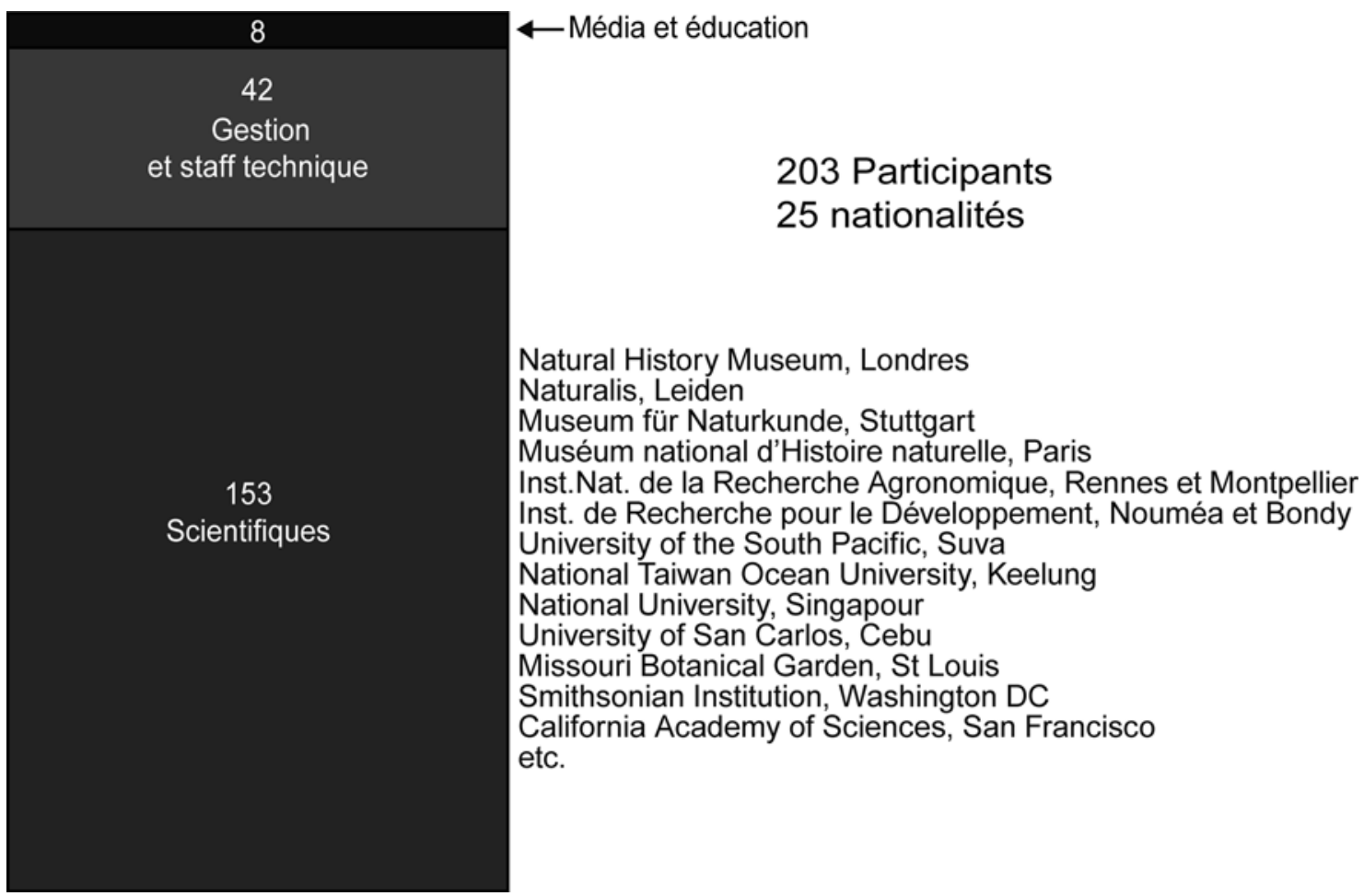

FiguRE 3. - Participants à l'expédition Santo 2006 : répartition par fonctions

du Pacifique avec des espèces nouvelles de poissons, de grillons ou de crevettes, est un résultat prévisible et attendu. Par contre, que le journal économique Les Échos consacre une demi-page à Santo 2006 avec le titre « Le retour des grandes expéditions naturalistes?» l'est beaucoup moins, de même que l'accueil reçu par le livre de Vincent Tardieu et Lise Barnéoud (Santo, les explorateurs de l'île planète) paru chez Belin ${ }^{5}$, et par le long métrage Santo, l'île planète, diffusé sur France 3 dans le cadre de l'émission Thalassa, puis rediffusé en deux parties sur la 5 . Un résultat espéré mais inattendu de Santo 2006 aura été de démontrer qu'il est possible d'intéresser le public à toute la biodiversité.

Quelles ont été, a posteriori, les raisons de ce succès ? Vis-à-vis d'un public avide, mais également saturé - voire blasé - d'images, la moisson de photographies numériques amassées pendant l'expédition constitue une véritable révélation de ce monde si mal connu. Ensuite, dans l'élaboration de l'expédition, nous avons tenté de placer l'inventaire de la biodiversité dans son contexte humain et de l'enraciner dans son histoire : paléoclimatologues, archéologues, dendrochronologues ont porté leur regard sur les derniers millénaires ; ethnologues, juristes et économistes de la biodiversité se sont intéressés aux perceptions de cette biodiversité ; enfin, journalistes, photo- graphes, illustrateurs, enseignants et cinéastes ont fait partager la vie de l'expédition avec le plus grand nombre, dans le pays hôte comme en France.

Ce changement d'échelle a facilité la mobilisation de ressources juridiques et le soutien des postes diplomatiques pour répondre aux changements dans les modes d'accès à la biodiversité. Au-delà de Santo, nous espérons avoir montré que la poursuite scientifiquement légitime des missions de recherche et de constitution de collections sur les faunes et les flores passe par le développement de pratiques institutionnelles, enracinées dans les sciences de l'Homme, pour négocier des conditions transparentes d'accès à la biodiversité, donner des garanties sur les procédures d'étude (gènes, molécules) qui respectent les engagements négociés, et apprendre à mettre en œuvre des volets de restitution et de partage des « avantages » en direction des pays du Sud.

Les taxonomistes publient leurs résultats sous forme de descriptions ponctuelles d'espèces nouvelles dans des articles qui paraissent dans des journaux très spécialisés lus par les seuls spécialistes (voir, par exemple, Chazeau, 1979 ; Kurahashi, 1982 ; Keith et al., 2007 ; West et al., 2007) et l'exploitation des résultats d'une grande expédition naturaliste n'est jamais terminée. Il paraît encore des résultats inédits de la Mangarevan 
Forêts, montagnes et rivières
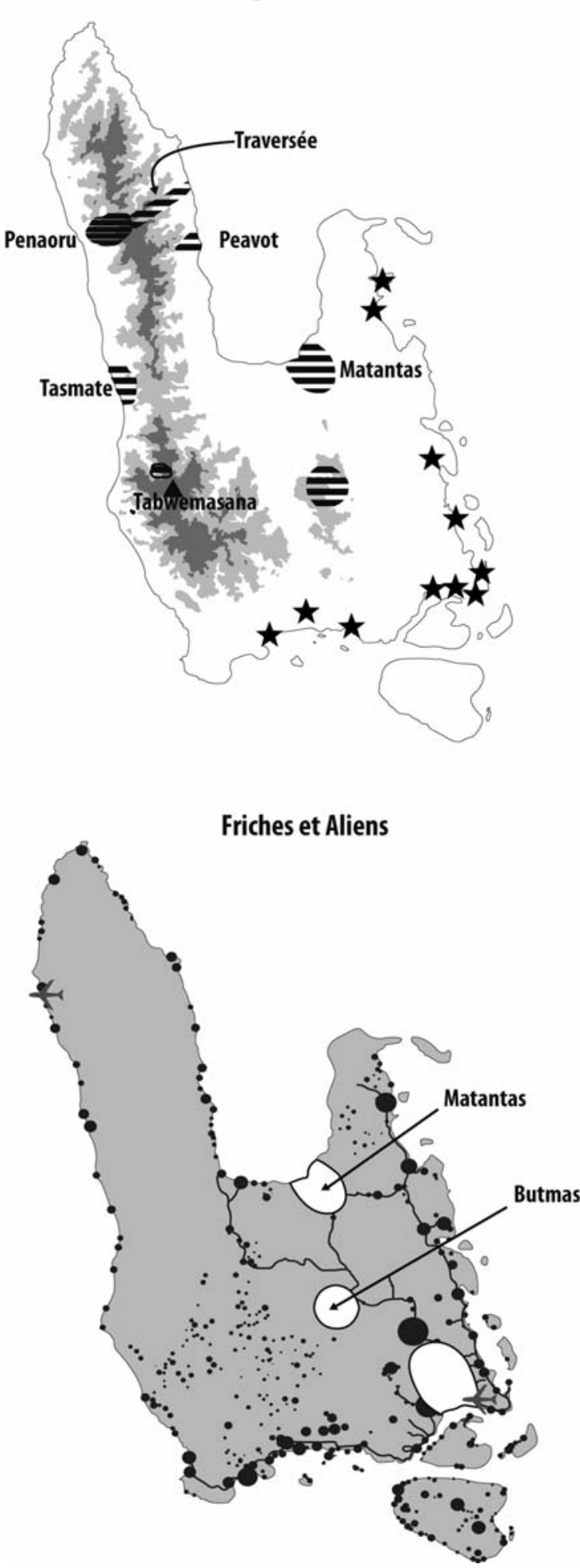
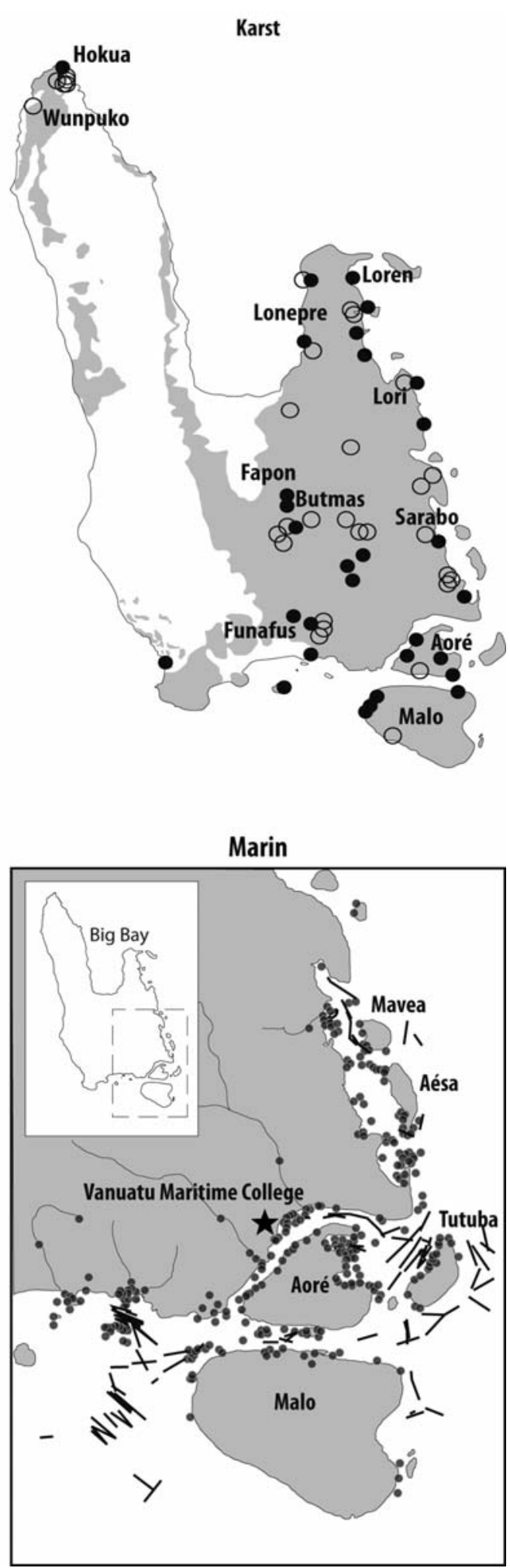

FIGURE 4. - Localisation dans l'île des sites d'activité des quatre modules

Forêts, montagnes, rivières. Hachures serrées : échantillonnage intensif ; hachures plus lâches : échantillonnage moins intensif ; étoiles : échantillonnages ponctuels.

Karst. Cercles pleins : grottes sèches; cercles vides : grottes avec aquifère.

Friches et aliens. Les trois sites d'activité : région de Luganville, Matantas et Butmas. Le fond de carte montre la densité de la

population (recensement 1988, la population de Luganville n'est pas représentée), les pistes et les terrains d'aviation.

Marin. Cercles pleins : sites de plongée et de prélèvements à marée basse ; segments : traits de chalutage ou de dragage. 
Expedition conduite par le Bishop Museum d'Honolulu en... 1934 ! Santo 2006 ne fera pas exception : il est probable qu'il continuera de paraître, dans vingt ou cinquante ans, des descriptions d'espèces collectées lors de l'expédition. Pour autant, il n'est pas nécessaire d'avoir identifié chaque gastéropode ou chaque diptère pour produire les résultats d'ensemble qui sont attendus par les non-spécialistes. Le Natural History of Santo, qui paraîtra en mai 2009, remplira cette fonction. En un peu plus de 500 pages, la géographie de l'île, les principaux milieux, l'originalité des faunes et des flores, seront présentés dans un langage scientifique accessible, aussi allégé que possible des terminologies de spécialistes. Ce sera le livre que nous aurions tous aimé avoir en arrivant à Santo et nous espérons que ce sera un livre qui inspirera d'autres explorateurs dans le Pacifique Sud.

\section{Remerciements}

Une expédition de la dimension de Santo 2006 n'a été possible que grâce au soutien de nombreux organismes, institutions et personnes, qu'il n'est malheureusement pas possible d'énumérer ici, mais qui figureront dans Natural History of Santo. Nous voudrions toutefois adresser particulièrement notre gratitude à Russell Nari et à Maxime Korman Carlot, au ministère des Terres, à Joël Path, à la province de Sanma, et à Bernard Sexe, pour la confiance qu'ils nous ont témoignée tout au long du projet. Les fondations, entreprises et organisations mentionnées dans la figure 1 ont assuré le financement. Les personnels du Centre technique de recherche en agronomie du Vanuatu (CTRAv), du Maritime College, les chefs coutumiers et l'ensemble des communautés d'Aoré, Butmas, Funafus, Malo, Natawa, Peavot, Penaoru, Tasmate, Vathe, Wunpoku et de bien d'autres villages qui ont accueilli les scientifiques de l'expédition avec un mélange de patience, de désintéressement et de curiosité amusée. Qu'ils sachent qu'ils ont laissé, chacun, leur empreinte dans notre mémoire.

\section{BIBLIOGRAPHIE}

Autheman Jean-Pierre et Jean-Paul Dethorey, 1999. Le passage de Vénus, 2 tomes, Paris, Dupuis.

Baillie J.E.M., C. Hilton-Taylor and S.N. Stuart (eds), 2004. 2004 IUCN Red List of threatened species. A global species assessment, Gland (Switzerland) and Cambridge (UK), IUCN, XXIV + $191 \mathrm{p}$.
Bouchet Philippe, 2007. L'insaisissable inventaire des espèces, Les Dossiers de La Recherche 28, pp. 4855.

Bougainville Louis Antoine (DE), 2006. Bougainville et ses compagnons autour du monde, réédition du « Voyage autour du monde, par la frégate du Roi la Boudeuse et la flûte l'Étoile », 1771, Paris, Imprimerie nationale.

Chazeau Jean, 1979. Sukunahikona prapawan, nouvelle espèce de Coccinellidae de Mélanésie, Bulletin de la Société entomologique de France 84, pp. 117121.

Domeny de RiEnzi G. L., 1836-1837. Océanie ou cinquième partie du monde. Revue géographique et ethnographique de la Malaisie, de la Micronésie, de la Polynésie et de la Mélanésie, 3 tomes, Paris, Firmin Didot.

DuboIs Alain, 2008. Handicap taxinomique et crise de la biodiversité : un nouveau paradigme pour la biologie au XXI ${ }^{\mathrm{e}}$ siècle, in D. Prat, A. Raynal-Roques et A. Roguenant (éds), Peut-on classer le vivant? Linné et la systématique aujourd'hui, Paris, Belin, pp. 141160.

Dumont D'URVILle Jules, 1834-1836. Voyage pittoresque autour du monde ou résumé général des voyages de découvertes, 2 tomes, Paris, L. Tenré.

Galipaud Jean-Christophe et Ian LiLley (éds.), 1999. Le Pacifique de 5000 à 2000 avant le présent : suppléments à l'histoire d'une colonisation, actes de la troisième conférence Lapita, Vanuatu, 31 juillet- 6 août 1996, Paris, IRD, 619 p.

GiAnGRANDE Adriana, 2003. Biodiversity, conservation and the "Taxonomic impediment", Aquatic Conservation 13 (5), pp. 451-459.

Gomez-Pompa Arturo, 2004. The role of biodiversity scientists in a troubled world. BioScience 54 (3), pp. 217-225.

Keast Allen and Scott E. Miller, 1996. The origin and evolution of Pacific Island biotas, New Guinea to Eastern Polynesia: patterns and processes, Amsterdam, SPB Academic Publishing, 531 p.

KeIth Philippe, Gérard Marquet and Ronald E. WATSON, 2007. Stiphodon kalfatak, a new species of freshwater goby from Vanuatu (Gobioidei: Sicydiinae), Cybium 31 (1), pp. 33-37.

KIRCH Patrick Vinton, 2000. On the road of the winds. An archaeological history of the Pacific Islands before European contact, Berkeley, University of California Press, 424 p.

KIRCH Patrick Vinton and Terry HunT (eds), 1997. Historical ecology in the Pacific Islands. Prehistoric environmental and landscape change, New Haven, Yale University Press, $331 \mathrm{p}$.

Koh L.P., R.R. Dunn, N.S. Sodhi, R.K. Colwell, H.C. Proctor and V.S. Smith, 2004. Species coextinctions and the biodiversity crisis, Science 305 (5690), pp. 1632-1634.

KuRAHASHI Hiromu, 1982. Blow flies from Vanuatu (New Hebrides), with descriptions of three new 
species of the genus Onesia (Diptera: Calliphoridae), Pacific Insects 24 (3-4), pp. 235-249.

LEAKEY Richard and R. LEWIN, 1995. The sixth extinction. Biodiversity and its survival, London, Orion Books, $271 \mathrm{p}$.

LewIS Beverley and Phillip CRIBB, 1989. Orchids of Vanuatu, Royal Botanic Gardens, Kew, 171 p.

Mead Jim, David Steadman, Stuart Bedford, Christopher BeLL and Matthew Spriggs, 2002. New extinct mekosuchine crocodile from Vanuatu, South Pacific. Copeia 2002 (3), pp. 632-641.

Mittermeier Russell, Patricio Robles Gil, Michael Hoffman, John PILgRIM, Thomas Brooks, Cristina GoetTSCH-MitTERMEIER, John LAMOREux and Gustavo A.B. DA Fonseca (eds), 2005. Hotspots revisited. Earth's biologically richest and most endangered terrestrial ecoregions, Conservation International / Cemex, University of Chicago Press, 391 p.

Mueller-Dombois Dieter and Raymond Fosberg, 1998. Vegetation of the tropical Pacific Islands, New York, Springer, $733 \mathrm{p}$.

New Tim R., 1995. An introduction to invertebrate conservation biology. Oxford, Oxford University Press, 194 p.

Noury Arnaud, 2005. Le reflet de l'âme Lapita. Essai d'interprétation des décors des poteries Lapita en Mélanesie et Polynésie occidentale (3200-2700 BP), Versailles, Noury éditions, $120 \mathrm{p}$.

O'Brian Patrick, 1997. Joseph Banks: A Life, Chicago, University of Chicago Press, 330 p.

O'BYrne Denis and David Harcombe, 1999. Vanuatu, $3^{\mathrm{e}}$ edition, Melbourne, Lonely Planet Publications, $304 \mathrm{p}$.
Pimm S.L., G.J. Rusell, J.L. Gittleman and T.M. Brooks, 1995. The future of biodiversity. Science 269, pp. 347-350.

Stattersfield Alison J., Michael J. Crosby, Adrian J. LONG and David C. WeGE, 1998. Endemic bird areas of the world: Priorities for biodiversity conservation, Birdlife International, University of California Press, $846 \mathrm{p}$.

Thomas C.D., A. Cameron, R.E. Green, M. BaKkeNeS, L.J. Beaumont, Y.C. Collingham, B.F.N. Erasmus, M. Ferreira De Siqueira, A. Grainger, L. Hannah, L. Hughes, B. Huntley, A.S. Van JaARsveld, G.F. Midgley, L. Miles, M.A. Ortega-Huerta, A. Townsend Peterson, O.L. Phillips and S.E. WiLliams, 2004. Extinction risk from climate change, Nature 427, pp. 145-148.

WCMC, 2000. Global Biodiversity: Earth's living resources in the $21^{\text {st }}$ century, Cambridge, World Conservation Press, $246 \mathrm{pp}$.

West John A., Giuseppe C. Zuccarello, Joseph L. Scott, Kathryn A. West and Susan LoISEAUX DE GoER, 2007. Pulvinus veneticus gen. et sp. nov. (Compsopogonales, Rhodophyta) from Vanuatu, Phycologia 46 (3), pp. 237-246.

WheEler Quentin D. and Joe Cracraft, 1997. Taxonomic preparedness: are we ready to meet the biodiversity challenge?, in M.L. Reaka-Kudla, D. Wilson and E.O. Wilson (éds.). Biodiversity II, Washington, D.C., Joseph Henry Press, pp. 435446. 
